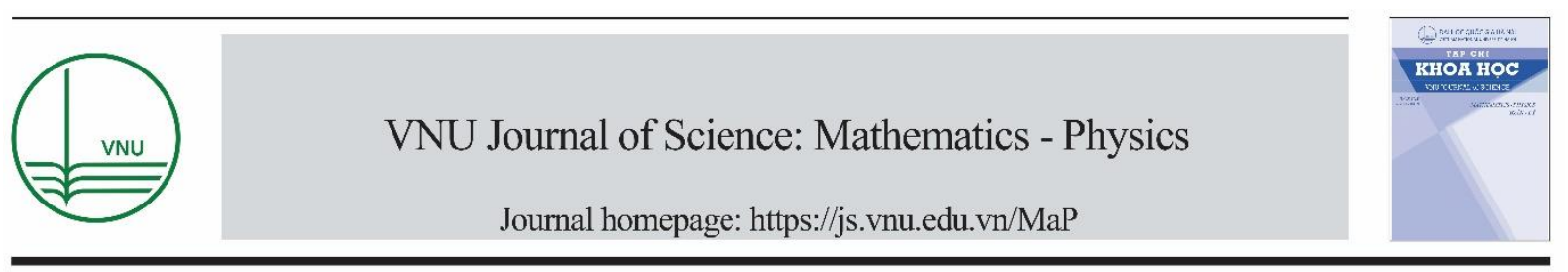

Original Article

\title{
Synthesis and Characterization of Silver Nanoparticles for Antibacterial Application against Bacillus Subtilis and Pseudomonas Aeruginosa
}

\author{
Pham Thi Thu Ha ${ }^{1}$, Vu Xuan Hoa ${ }^{1, *}$, Trinh Dinh Kha ${ }^{1,2}$, Nguyen Dac Dien ${ }^{3}$, \\ Luong Duy Thanh ${ }^{2}$, Nguyen Quang Hung ${ }^{4}, 5$, Luong Van Luyen ${ }^{6}$ \\ ${ }^{I}$ TNU University of Sciences, Tan Thinh, Thai Nguyen, Vietnam \\ ${ }^{2}$ Thuyloi University, 175 Tay Son, Dong Da, Hanoi, Vietnam \\ ${ }^{3}$ Vietnam Trade Union University, 169 Tay Son, Dong Da, Hanoi, Vietnam \\ ${ }^{4}$ Institute of Fundamental and Applied Sciences, Duy Tan University, Ho Chi Minh City, Vietnam \\ ${ }^{5}$ Faculty of Natural Sciences, Duy Tan University, Da Nang City, Vietnam \\ ${ }^{6}$ Vung Cao Viet Bac High School, Quyet Thang, Thai Nguyen, Vietnam
}

Received 04 August 2020

Revised 03 October 2020; Accepted 20 October 2020

\begin{abstract}
In this study, the stable silver nanoparticles (AgNPs) were synthesized by reducing silver nitrate (AgNO3) using trisodium citrate (TSC). The product was characterized by Ultraviolet-Visible spectroscopy, Fourier-transform infrared spectroscopy (FTIR), transmission electron microscopy (TEM) and X-ray diffraction analysis (XRD). UV-Vis spectrum showed a peak around $420 \mathrm{~nm}$. TEM analysis revealed the homogeneity in the size of AgNPs $(35-45 \mathrm{~nm})$, well-dispersed quasi-spherical in water. The prepared AgNPs exhibited high antibacterial activity against Bacillus subtilis and Pseudomonas aeruginosa bacteria. The average zones of inhibition were $20 \mathrm{~mm}$ and $17 \mathrm{~mm}$ for Pseudomonas aeruginosa and Bacillus subtilis bacteria, respectively. The inhibition zone of AgNPs was also compared to the reference antibiotics drugs such as ampicillin and natamycin. This research exhibits an efficient and eco-friendly synthesis of silver nanoparticles with potent antimicrobial and antibacterial performance.

Keywords: Silver nanoparticles, chemical synthesis, antibacterial activity, antimicrobial agent, cell inhibition.
\end{abstract}

\footnotetext{
* Corresponding author

Email address: hoavx@tnus.edu.vn
}

https://doi.org/10.25073/2588-1124/vnumap.4591 


\section{Introduction}

The development of new effective antimicrobial reagents based on metal nanomaterials has replaced the conventional antibiotics due to emergence of resistant microbes. Silver nanoparticles (AgNPs) have long been known to have strong antibacterial effects as well as antimicrobial activities $[1,2]$ and could be considered as a potential alternative for existing drugs. AgNPs have also wide applications in the biomedical and environmental fields such as biological labeling, drug delivery, cancer therapy, molecular imaging, diagnostics, textile, catalysis, photography, electronics and food industry [3-6]. In one of our previous works [7], the antibacterial activity against Escherichia coli and Staphylococcus aureus of silver nanoparticles was reported, where the colloidal silver solution was successfully synthesized in a wet chemical process using silver nitrate $\left(\mathrm{AgNO}_{3}\right)$, sodium borohydride $\left(\mathrm{NaBH}_{4}\right)$ and starch as starting agents. It has been proven that AgNPs exhibited an excellent antibacterial activity against Gram-negative bacteria Escherichia coli (E. coli) and Gram-positive Staphylococcus aureus (S. aureus). It is also reported that the average inhibition zone of AgNPs was $7.7 \mathrm{~mm}$ for Escherichia coli and $7 \mathrm{~mm}$ for Staphylococcus aureus bacteria. In this study, AgNPs were synthesized from silver nitrate $\left(\mathrm{AgNO}_{3}\right)$ and trisodium citrate dihydrate (TSC) and the average inhibition zones of AgNPs were found to be $17 \mathrm{~mm}$ for Bacillus subtilis and $20 \mathrm{~mm}$ for Pseudomonas aeruginosa bacteria. Several proposals have been developed to explain the inhibitory effects of AgNPs on bacteria. It is believed that reaction of heavy metal with proteins leads to the inactivation of the proteins in bacteria [8] and interaction between silver atoms with the sulfur-containing proteins plays an essential role in cellular damage [9]. It is revealed that the silver atoms can destroy microorganisms completely through oxidative catalysis mechanism [10]. Experimental results may provide useful information for understanding the antibacterial effects and inhibitive process of silver atoms although the mechanism of antimicrobial effects of silver atoms on bacteria is complicated. It is noticeable that AgNPs are non-toxic to animal cells but highly toxic to bacteria, so they are very promising antibacterial systems.

\section{Materials and Methods}

Silver nitrate $\left(\mathrm{AgNO}_{3}, 99.8 \%\right)$, trisodium citrate dihydrate ( $\left.\mathrm{TSC}, \mathrm{Na}_{3} \mathrm{C}_{6} \mathrm{H}_{5} \mathrm{O}_{7} .2 \mathrm{H}_{2} \mathrm{O}, 99 \%\right)$ supplied by Sigma-Aldrich were of analytical grade and used as received. Deionized water prepared with a MilliQ water purification system was used to prepare the solutions and to clean all glassware and experimental tools. The synthesis process of colloidal silver was carried out as follows. Firstly, $\mathrm{AgNO}_{3}$ was dissolved in the deionized water to obtain $30 \mathrm{ml}$ of $1 \mathrm{mM} \mathrm{AgNO}_{3}$ solution. Then, it was heated until boiling. After that, $5 \mathrm{ml}$ of $30 \mathrm{mM}$ TSC solution was added drop-wise to the $\mathrm{AgNO}_{3}$ solution under vigorous stirring. The molar ratio of $\mathrm{TSC} / \mathrm{AgNO}_{3}$ was $5: 1$, the volume ratio of $\mathrm{TSC} / \mathrm{AgNO}_{3}$ was 1:6 and injection time was $6 \mathrm{~min}$. The reaction was maintained at the boiling status for $14 \mathrm{~min}$ in the dark to avoid direct exposure to the natural light. After the reaction was completed, the stock solution was cooled down to the room temperature. The chemical reaction was the reduction of silver ions by TSC:

$$
4 \mathrm{AgNO}_{3}+\mathrm{Na}_{3} \mathrm{C}_{6} \mathrm{H}_{5} \mathrm{O}_{7}+2 \mathrm{H}_{2} \mathrm{O} \rightarrow 4 \mathrm{Ag} \downarrow+\mathrm{H}_{3} \mathrm{C}_{6} \mathrm{H}_{5} \mathrm{O}_{7}+3 \mathrm{NaNO}_{3}+\mathrm{HNO}_{3}+\mathrm{O}_{2} \uparrow
$$

The change of the color of the reaction solution from transparent to pale yellow was an indicator for the reduction of the $\mathrm{Ag}^{+}$ions into metallic silver. UV-Vis spectrophotometer (HITACHI-U2900, Japan) was used to characterize the structure of silver nanoparticles (wavelength of 250-600 nm). Transmission electron microscopy (TEM, JEM-1010 JEOL) with an electron kinetic energy of $80 \mathrm{keV}$ was used to investigate the morphology and size of the products. The size-distribution profile of AgNPs was studied by using dynamic light scattering (DLS) measurements conducted with a particle size analyzer (PSA, Delta Nano C, Beckman). The Fourier-Transform Infrared (FTIR) spectra recorded on the Spectrum 
FTIR Affinity-1S (SHIMADZU) in the range of $500-4000 \mathrm{~cm}^{-1}$ at a scan speed of $16 \mathrm{~cm} / \mathrm{s}$ was used to detect the functional groups on the AgNPs. X-ray diffraction pattern was performed using an X-ray diffractometer (Siemens D5005) with the wavelength of $\mathrm{Cu}-\mathrm{K}_{\alpha}$ radiation of $\lambda=1.5417 \AA$ to analyze the phase structure and exact material identification of samples. Bacillus subtilis and Pseudomonas aeruginosa bacteria were selected as the test micro-organisms to evaluate the antibacterial activity of AgNPs. All cultures were procured from Thai Nguyen University of Sciences (Vietnam) and were maintained at $4{ }^{\circ} \mathrm{C}$. A sterile agar plate was perforated to form a well with the diameter of $6 \mathrm{~mm}$. The antibacterial performance of AgNPs was checked by agar well diffusion assay. $15 \mathrm{ml}$ of nutrient medium was poured onto the plate then allowed to solidify for $5 \mathrm{~min}$. After that, $100 \mu \mathrm{l}$ of inoculum suspension containing bacteria was spread uniformly on the plate and allowed to dry for $5 \mathrm{~min}$. Finally, $70 \mu \mathrm{l}$ of AgNPs colloidal solution was loaded into the well on the plate. After incubating at $37^{\circ} \mathrm{C}$ for $24 \mathrm{~h}$, the diameter of inhibition zone was measured and recorded as a mean value of the triplicate experiment.

\section{Results and Discussion}

The growth mechanism of AgNPs obtained by the oxidation-reduction reaction between sodium citrate and silver nitrate was described by the diagram in Figure 1a. The metallic silver $\left(\mathrm{Ag}^{0}\right)$ was first formed from the $\mathrm{Ag}^{+}$reduction by citrate. The $\mathrm{Ag}$ atoms aggregated to form silver cluster and the initial seeds began to grow via the Ostwald ripening, in which the larger particles grew at the expense of the smaller ones. The UV-Vis absorption spectrum of the synthesized AgNPs is shown in Figure 1b. It can be seen that the plasmon absorption band is located at $420 \mathrm{~nm}$ with the value of the absorbance intensity of about 2.83 (arbitrary unit) corresponding to the typical characteristic surface plasmon resonance (SPR) of the spherical AgNPs. In addition, the spectrum has only one peak, indicating that the particles are mainly spherical with small size and regular distribution. This result is in good agreement with previous findings, that the number of surface plasmon resonance (SPR) peaks increases as the symmetry of particles decreases [11]. The full width at half maximum (FWHM) is of $90 \mathrm{~nm}$. The SPR peak is narrow showing the reductive process of $\mathrm{Ag}^{+}$ions was completed and obtained AgNPs were uniform.

TEM image of the AgNPs and their size distribution is shown in Figure 2a and Figure $2 b$, respectively. It is clearly revealed that AgNPs have a uniform spherical shape of about $35-45 \mathrm{~nm}$, the average size is $40 \mathrm{~nm}$ in diameter. Figure 3 shows the color of the colloidal AgNPs solution at different reaction times. The color changed from translucent to darker when the reaction time increased.
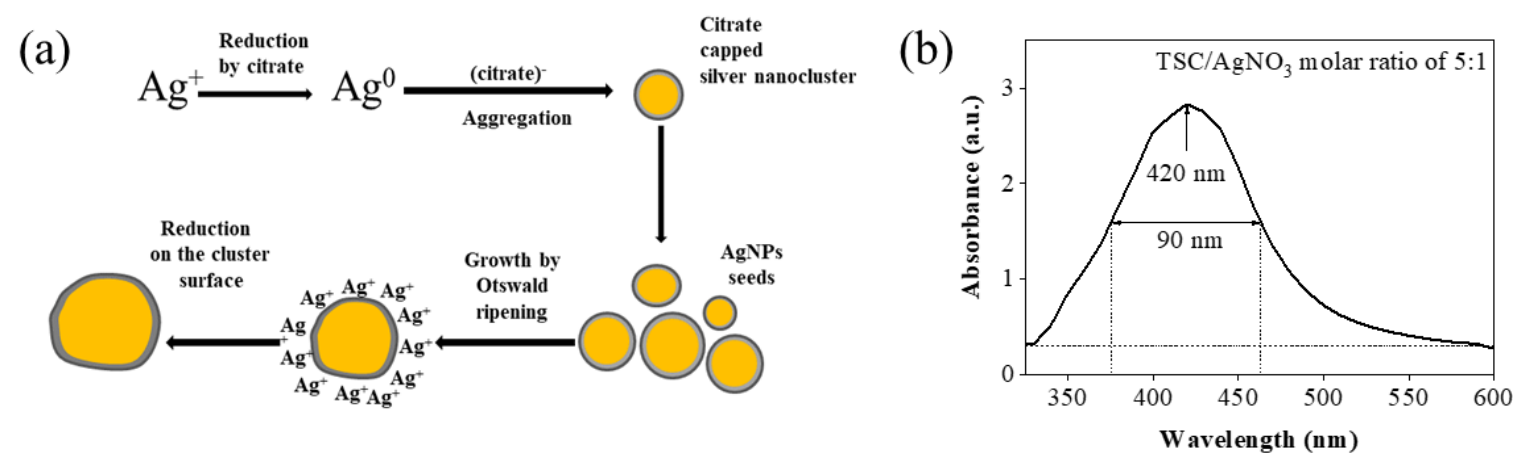

Figure 1. (a) Growth mechanism, (b) UV-Vis absorption spectrum of the AgNPs. 

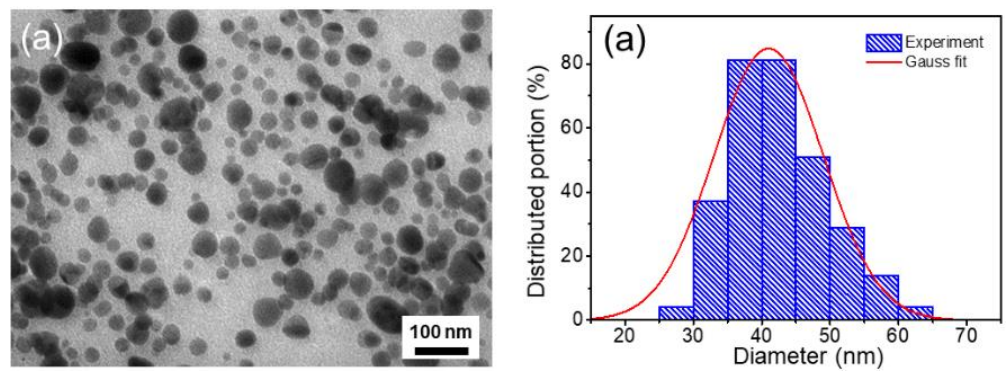

Figure 2. (a) TEM image, (b) The size distribution of AgNPs.
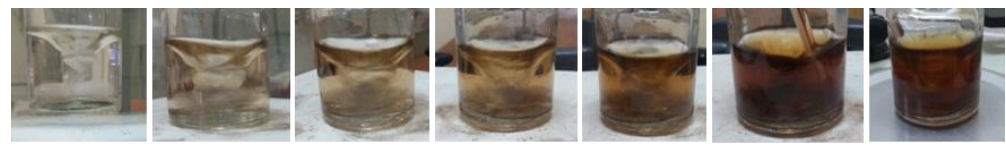

$4 \min$

$8 \mathrm{~min}$

$14 \mathrm{~min}$

$20 \mathrm{~min}$

$25 \mathrm{~min}$

$32 \mathrm{~min}$

$42 \min$

Figure 3. The change of the AgNPs solution color according to reaction time.
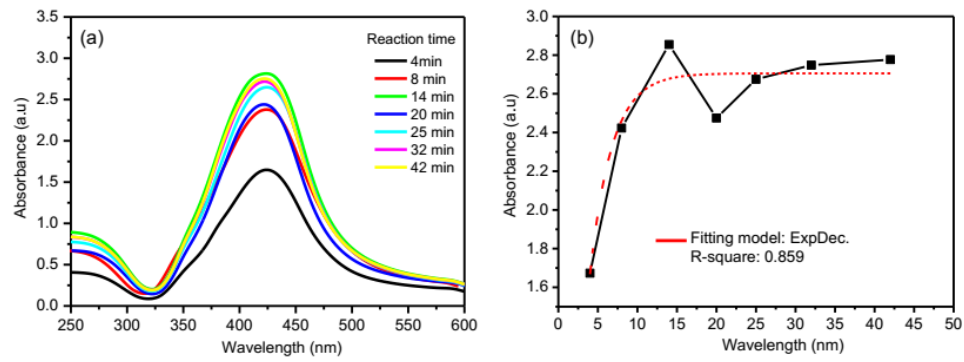

Figure 4. (a) The UV-Vis absorbance spectra of AgNPs prepared for different reaction time,

(b) Dependence of absorption peak intensity on the reaction time.

The UV-Vis absorption spectra of the samples synthesized for different reacting times are shown in Figure 4a. The intensity of the surface plasmon band increases quasi linearly with respect to the reaction time in the first $14 \mathrm{~min}$ and remains unchanged when the reaction time is prolonged to $42 \mathrm{~min}$ (Figure 4b). All SPR peaks typically located between $420-430 \mathrm{~nm}$, characteristic of silver nanoparticles [12]. This observation shows that the nucleation and growth reaction were completed after 14 min.

Figure 5a displays the XRD pattern of the synthesized AgNPs. Four characteristic peaks were observed at $37.68^{\circ}, 44.56^{\circ}, 65.07^{\circ}$ and $76.62^{\circ}$ corresponding to the diffraction of (111), (200), (220) and (311) crystalline planes, respectively, which can be readily indexed to a face-centered cubic (FCC) lattice structure of silver (JCPDS No.04-0783) [13]. Figure 5b depicts the FTIR spectrum of AgNPs. The strong absorption peaks at 1590,1398 and $1372 \mathrm{~cm}^{-1}$ wavenumbers represent the presence of $\mathrm{NO}_{2}$ group coming from the $\mathrm{AgNO}_{3}$ solution. The peaks at 3413 and $2926 \mathrm{~cm}^{-1}$ associate with the $\mathrm{OH}$ functional group. The peak at $1058 \mathrm{~cm}^{-1}$ was due to stretching of C-OH binding [2].

It is known that if AgNPs are unstable and aggregated, the plasmon peak intensity at $420 \mathrm{~nm}$ will decrease. So the UV-Vis spectroscopy can be used for monitoring the stability of AgNPs and the result is shown in Figure 6. It can be seen that the plasmon peak intensity slightly decreased after more than 40 days, which indicated the stability of the sample over time. Therefore, the sample could be kept and used for antibacterial activity for a long time. 

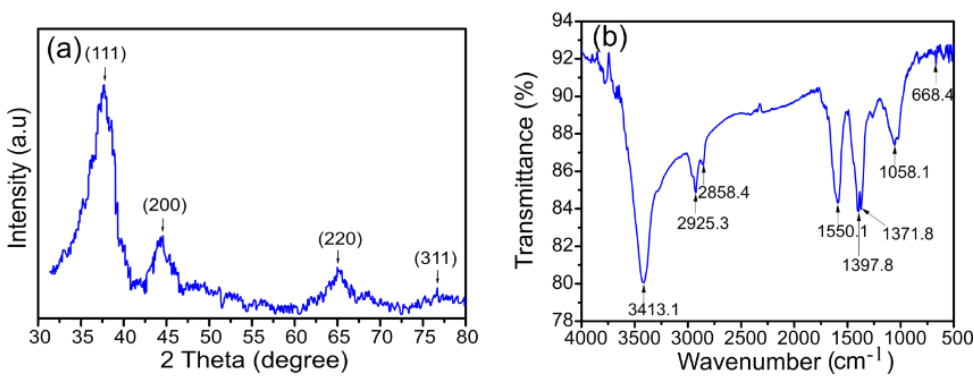

Figure 5. (a) XRD pattern, (b) FTIR spectrum of silver nanoparticles.

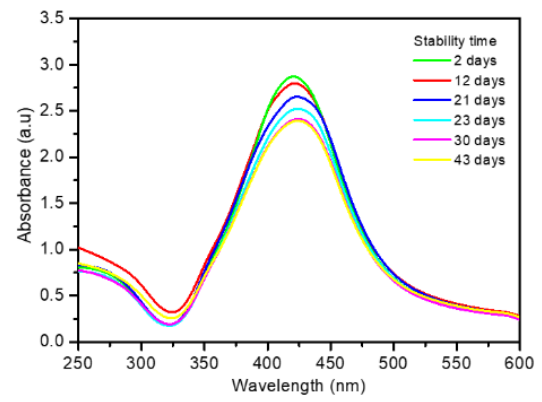

Figure 6. UV-Vis spectra of colloidal AgNPs solution recorded after 43 days.

It is known that the antibacterial effect of AgNPs depends on several parameters such as the size, shape and the surface charge of the particles. AgNPs can be attached to the surface of the bacterial cell membrane by interacting with the sulfur-containing proteins, disturbing permeability and respiration function of the cell, resulting in cell death [14]. AgNPs can also penetrate the cell wall into the bacteria, causing the deterioration of the membrane and cell destruction [15]. AgNPs may interact with the thiol group compound in the respiratory enzyme of bacterial cell, thus inhibiting the respiration process in the bacteria [14]. AgNPs can condense the DNA and prevent the replication of the DNA as well as the reproduction of the cells [16]. The silver ions released from AgNPs may penetrate inside the cell and interact with phosphorus in protein and DNA of bacteria, resulting in the loss of the cell viability, and ultimately damaging the cell.
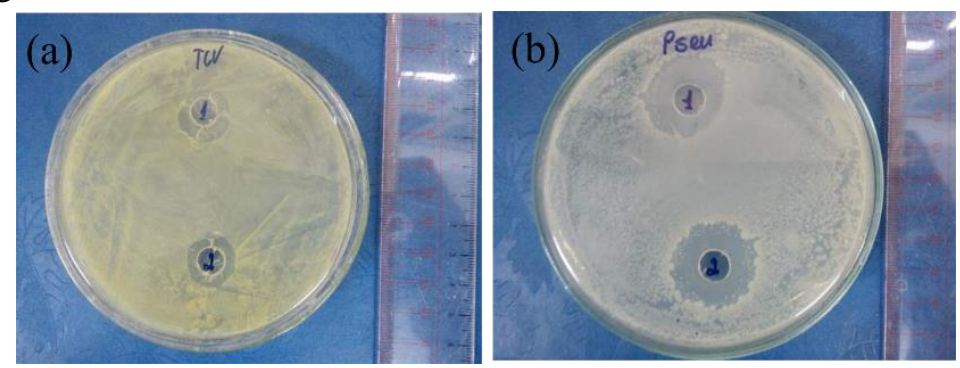

Figure 7. Antibacterial activity of AgNPs against (a) Bacillus subtilis and (b) Pseudomonas aeruginosa.

Figure 7 presents the antibacterial activity of AgNPs. The zone of inhibition of $20 \mathrm{~mm}$ was observed in the Pseudomonas aeruginosa bacteria and the zone of $17 \mathrm{~mm}$ was observed in the Bacillus subtilis. The inhibition zone of Pseudomonas aeruginosa was found to be wider than that of Escherichia coli (10 
$\mathrm{mm})$. Similarly, the inhibition zone of Bacillus subtilis was found to be wider than that of Staphylococcus aureus $(12 \mathrm{~mm})$ [7]. The inhibition zone of AgNPs was also compared against the reference antibiotics drugs containing ampicillin. The results show that the antibacterial activity for Bacillus subtilis of the $70 \mu \mathrm{l}$ of colloidal AgNPs solution was approximately equal to $90 \%$ that of 0.875 mg ampicillin antibiotics, and the antibacterial activity for Pseudomonas aeruginosa of AgNPs and ampicillin was almost the same.

\section{Conclusions}

We have used a method of reduction $\mathrm{Ag}^{+}$to $\mathrm{Ag}^{0}$ by TSC for synthesizing uniform spherical AgNPs with average diameter of $40 \mathrm{~nm}$ in size and stable for long term (over $40 \mathrm{~h}$ ). The obtained results show that the synthesized AgNPs exhibited an excellent antimicrobial activity against the microorganism disease, such as Bacillus subtilis and Pseudomonas aeruginosa, known as multidrug resistant and hazardous bacteria. The average zones of inhibition for Bacillus subtilis and Pseudomonas aeruginosa were of $17 \mathrm{~mm}$ and $20 \mathrm{~mm}$, respectively. Such AgNPs constitute a potential candidate for antibacterial applications.

\section{Acknowledgements}

This research is funded by Vietnam National Foundation for Science and Technology Development (NAFOSTED) under Grant 103.03-2020.43.

\section{References}

[1] Q. L. Feng, J. Wu, G. Q. Chen, F. Z. Cui, T. N. Kim, J. O. Kim, A Mechanistic Study of The Antibacterial Effect of Silver Ions on Escherichia Coli and Staphylococcus Aureus, J. Biomed. Mater. Res, Vol. 52, No. 4, 2000 , pp. 662-668, https://doi.org/10.1002/1097-4636(20001215)52:4\%3C662::aid-jbm10\%3E3.0.co;2-3.

[2] D. Bhatia, A. Mittal, D. K. Malik, Antimicrobial Activity of PVP Coated Silver Nanoparticles Synthesized by Lysinibacillus Varians, 3 Biotech, Vol. 6, No. 196, 2016, pp. 1-8, https://doi.org/10.1007/s13205-016-0514-7

[3] N. G. Mlalila, H. S. Swai, A. Hilonga, D. M. Kadam, Antimicrobial Dependence of Silver Nanoparticles on Surface Plasmon Resonance Bands Against Escherichia Coli, Nanotechnol. Sci. Appl, Vol. 2017, No. 10, 2016, pp. 1-9, https://doi.org/10.2147/NSA.S123681.

[4] X. F. Zhang, Z. G. Liu, W. Shen, S. Gurunathan, Silver Nanoparticles: Synthesis, Characterization, Properties, Applications, and Therapeutic Approaches, Int. J. Mol. Sci, Vol. 17, No. 9, 2016, pp. 1534, https://doi.org/10.3390/ijms17091534.

[5] A. J. Haes, R. P. V. Duyne, Preliminary Studies and Potential Applications of Localized Surface Plasmon Resonance Spectroscopy in Medical Diagnostics, Expert Rev. Mol. Diagn, Vol, 4, No. 4, 2004, pp. 527-537, https://doi.org/10.1586/14737159.4.4.527.

[6] M. Rai, A. Yadav, A. Gade, Silver Nanoparticles as A New Generation of Antimicrobials, Bio. Adv, Vol. 27 , No. 1, 2018, pp. 76-83, https://doi.org/10.1016/j.biotechadv.2008.09.002.

[7] X. H. Vu, T. T. T. Duong, T. T. H. Pham, D. K. Trinh, X. H. Nguyen, V. S. Dang, Synthesis and Study of Silver Nanoparticles for Antibacterial Activity Against Escherichia Coli and Staphylococcus Aureus, Adv. Nat. Sci. Nanosci. Nanotechnol, Vol. 9, No. 2, 2018, pp. 025019, https://doi.org/10.1088/2043-6254/aac58f.

[8] D. L. Nelson, M. M. Cox, Principles of Biochemistry, W.H. Freeman and Company, New York, USA, 2017.

[9] L. Kvitek, A. Panacek, J. Soukupova, M. Kolar, R. Vecerova, R. Prucek, M. Holecova, R. Zboril, Effect of Surfactants and Polymers on Stability and Antibacterial Activity of Silver Nanoparticles (NPs), J. Phys. Chem. C, Vol. 112, No. 15, 2008, pp. 5825-5834, https://doi.org/10.1021/jp711616v. 
[10] R. L. Davies, S. F. Etris, The Development and Functions of Silver in Water Purification and Disease Control, Catal. Today, Vol. 36, No. 1, 1997, pp. 107-114, https://doi.org/10.1016/S0920-5861\%2896\%2900203-9.

[11] B. Wiley, Y. Sun, B. Mayers, Y. Xia, Shape-Controlled Synthesis of Metal Nanostructures: The Case of Silver, Chem. A Eur. J, Vol. 11, No. 2, 2005 , pp. 454-463, https://doi.org/10.1002/chem.200400927.

[12] J. K. Patra, K. H. Baek, Antibacterial Activity and Synergistic Antibacterial Potential of Biosynthesized Silver Nanoparticles Against Foodborne Pathogenic Bacteria Along With its Anticandidal and Antioxidant Effects, Front. Microbiol, Vol. 8, No. 167, 2017, pp. 1-14, https://doi.org/10.3389/fmicb.2017.00167.

[13] P. Prakash, P. Gnanaprakasam, R. Emmanuel, S. Arokiyaraj, M. Saravanan, Green Synthesis of Silver Nanoparticles From Leaf Extract of Mimusops Elengi, Linn. for Enhanced Antibacterial Activity Against Multi Drug Resistant Clinical Isolates, Colloids Surf. B: Biointerfaces, Vol. 108, 2013, pp. 255-259, https://doi.org/10.1016/j.colsurfb.2013.03.017.

[14] W. R. Li, X. B. Xie, Q. S. Shi, H. Y. Zeng, Y. S. Ou-Yang, Y. B. Chen, Antibacterial Activity and Mechanism of Silver Nanoparticles on Escherichia Coli, Appl. Microbiol. Biotechnol, Vol. 85, 2010, pp. 1115-1122, https://doi.org/10.1007/s00253-009-2159-5.

[15] J. R. Morones, J. L. Elechiguerra, A. Camacho, K. Holt, J. B. Kouri, J. T. Ramirez, M. J. Yacaman, The Bactericidal Effect of Silver Nanoparticles, Nanotechnol, Vol. 16, No. 10, 2005, pp. 2346-2353, http://dx.doi.org/10.1088/09574484/16/10/059.

[16] M. Chen, Z. Yang, H. Wu, X. Pan, X. Xie, C. Wu, Antimicrobial Activity and The Mechanism of Silver Nanoparticle Thermosensitive Gel, Int. J. Nanomed, Vol. 6, 2011, pp. 2873-2877, https://doi.org/10.2147/IJN.S23945. 